\title{
Seeing is believing but quantifying is deciding
}

\section{Cátia DF Lopes 1,2,3, Maria Gomez-Lazaro1,2,4, Ana Paula Pêgo',2,5,6}

${ }^{1}$ INEB - Instituto de Engenharia Biomédica, Universidade do Porto, Rua do Campo Alegre 823, 4150180 Porto, Portugal.

${ }^{2} i_{3} S$ - Instituto de Investigação e Inovação em Saúde, Universidade do Porto, Portugal. 3FMUP - Faculdade de Medicina da Universidade do Porto, Porto, Portugal.

4b.IMAGE - Bioimaging Centre for Biomaterials and Regenerative Therapies, INEB, Universidade do Porto, Porto, Portugal.

5FEUP - Faculdade de Engenharia da Universidade do Porto, Porto, Portugal.

${ }^{6}$ ICBAS - Instituto de Ciências Biomédicas Abel Salazar, Universidade do Porto, Porto, Portugal.

*Author for correspondence: E-mail Address: apego@ineb.up.pt

Originally published in Nanomedicine (Lond). 2015;10(15):2307-10. doi: 10.2217/nnm.15.101. Epub 2015 Jul 31.

Keywords: bioimaging • imaging flow cytometry $\bullet$ intracellular trafficking $\bullet$ microfluidics $\bullet$ nanomedicine $\bullet$ nanoparticles $\bullet$ quantitative image analysis $\bullet$ tissue engineering

Drug and nucleic acid delivery by means of nanoparticle vectorization is a buzzing field in nanomedicine, with the ultimate goal being the achievement of a therapeutic outcome supported by an efficient delivery to the targeted cell population that does not compromise safety issues. Despite all the hype and progress that already spans several decades, still few strategies overcame the valley of death and reached the bedside. Many of the proposed systems have successfully conquered a number of hurdles in vitro and/or in pre-clinical animal models, nonetheless have failed in relevant clinical context. How can we (im)prove the efficiency and safety of nanoparticle delivery throughout the organism? Most of the work done to answer this central question is based on the evaluation of the interaction of nanoparticles with cells and tissues, mostly focusing on increasing nanoparticle internalization efficiency, and outcome assessment. It is well established that by tailoring the nanoparticle physicochemical properties such as size, shape, surface charge and composition, one can tune cell-nanoparticle interaction and uptake pathways. However, generalization of the assumptions related to the nanoparticles and their internalization pathways is dangerous since there are numerous exceptions to the proposed 'rules' [1]. On the other end of the process, by evaluating the outcome of nanoparticle administration, we can infer about the therapeutic efficiency of the proposed system and, at times, address safety issues. But the intracellular processes that occur inbetween nanoparticle cellular entry and biological response remain, at large, a black box. Additionally, few studies have examined the intracellular process of vector degradation or excretion $[\underline{2}, 3]$. In other words, in most cases neither we know the exact pathway and fate of the nanoparticles upon cellular entry, nor if their intracellular distribution is related with beneficial or adverse effects to the cell other than the expected outcome. Moreover, the information of the number of nanoparticles 
that reach a cell, as well as the number of nanoparticles that in fact efficiently deliver its cargo is, for the large majority of systems, unknown. Should one decide on the future of a proposed vector material or nanoparticle system solely based on such intangible information?

\section{Making visible the invisible}

Classical bioimaging methods applied in the study of nanoparticles in a cellular context are based on the fluorescent labeling of the nanoparticles, as well as of particular intracellular compartments, and the use of fluorescence microscopy, mostly laser scanning confocal microscopy, combined with flow cytometry [3]. These techniques can be complemented with electron transmission microscopy when the vectors have specific electron-optical contrast features or can be labeled with contrast agents [4]. However, the information that can be extracted from these analyses is incomplete. Confocal microscopy is widely used but the resolution limit is an obstacle when imaging particles below 100 $\mathrm{nm}$. Therefore, most of the studies are based on the analysis of nanoparticle-loaded vesicles inside the cell. In turn, electron microscopy allows for the imaging of nanoparticles independently of their size but to acquire enough quantitative data for statistical analysis is laborious and time consuming, hindering the possibility of a high-throughput evaluation. Although highly quantitative information can be obtained by flow cytometry, there is no spatial resolution and one cannot define the intracellular position of a particle nor distinguish nanoparticle cellular association from internalization. Currently alternative methodologies are being explored to address such unresolved questions, including: spinning disk confocal microscopy to study nanoparticle uptake kinetics and intracellular movement, taking advantage of reduced multichannel acquisition times [5]; atomic force microscopy to measure the force and/or specificity of the interaction between nanoparticles and the plasma membrane $\left[\boldsymbol{6}_{,}\right]$]; fluorescence correlation spectroscopy and raster image correlation spectroscopy, which can be used to obtain the hydrodynamic radii of the nanoparticles when internalized as well as kinetic parameters such as nanoparticle the intracellular diffusion coefficient [8] and super-resolution systems to overcome the limitation of the optical resolution of a regular confocal microscope [9], though imaging depth is still a constraint when working with cells growing on $3 \mathrm{D}$ scaffolds or even tissues [10]. To increase the imaging depth the use of multiphoton microscopy is an alternative. Other solutions take advantage of combining different technologies such as correlative light and electron microscopy that relates the ${ }_{3} \mathrm{D}$ distribution information obtained by confocal microscopy with the image resolution of the electron microscope for the analysis of single particles versus aggregates/agglomerates [11] and tip-enhanced Raman spectroscopy that allows a lateral resolution down to $10-20 \mathrm{~nm}$ giving chemical information that might be valuable to study also nanoparticle stability. Nevertheless, for quantification purposes all these techniques are quite time consuming and high-throughput platforms are emerging for a better quantification of internalization characteristics. In this direction, a promising solution is imaging flow cytometry, which combines the statistical strength of flow cytometry with image acquisition of every event making it possible to differentiate internalized from adsorbed nanoparticles $[12,13]$ and even explore intracellular localization.

All these techniques are normally used in combination with specific intracellular labels to address the nanoparticle intracellular fate or trafficking kinetics. To unravel the nanoparticle intracellular localization the conventional analyses focus mainly on the quantification of nanoparticle colocalization with specific intracellular organelles involved in the different internalization pathways, as nanoparticle internalization either occurs by endocytic mechanisms or passive internalization (rare event). Additionally, one can use pharmacological inhibitors to interrupt a certain intracellular 
pathway, as well as delete or express a mutant of a specific gene involved in the cellular uptake machinery. These strategies have proven to be very valuable but care must be taken when interpreting the obtained results. Available intracellular markers are not always exclusive for a specific organelle, since the endocytic pathway is a dynamic and continuous set of organelles that arise from a maturation process. Consequently, some membrane proteins used as markers are common between them [14]. Many of the available inhibitors lack specificity, blocking more than one pathway and are cytotoxic at the functional concentrations [15]. Last but not least, the nanoparticles specific entry route does not always determine their postinternalization fate and intracellular location [16]. Complimentary biochemical methods are used but also these rely on the use of specific biomarkers. Consequently, in parallel to the advance of new imaging technologies and modalities, several tools have been put forward to contribute to the knowledge of mechanism of nanoparticlemediated delivery. Fluorescent protein-based tools are now available to study nanoparticle properties, cellular uptake, subcellular trafficking, diffusion and stability inside the cell. Classically, one labels the vector and the cargo with different fluorophores to allow their tracking inside the cell [17]. A variation of this is the use of specific fluorophores for fluorescence resonance energy transfer that can capture nanoparticle intracellular dynamics [18]. Examples of other tools include: the use of fluorescent probes that are only active in a specific environment, that is, the reducing environment of the cell cytoplasm [19] or at a certain $\mathrm{pH}$ [20]; the application of fluorescence recovery after photobleaching to track nanoparticle dynamic properties inside the cell [8] and timer fluorescent proteins for the quantification and study of the outcome kinetics [21].

\section{Chip of reality}

While still many challenges remain ahead, several studies have already contributed to a better understanding of nanoparticle intracellular fate and to unravel a number of bottlenecks to the processes of nanoparticle-based delivery. Most of these have been based on in vitro set-ups. Resolution and lack of sensitivity of the current in vivo bioimaging tools remain an obstacle. Therefore, the knowledge of nanoparticle behavior at the whole body scale is still limited to the evaluation of overall biodistribution/accumulation in key organs, time of clearance, inflammatory reaction and associated toxicity.

In this context, current efforts are being drawn to the finding of alternative methods to animal experimentation, with the development of robust and predictive in vitro culture platforms that can more realistically mimic the in vivo conditions. Ideally, these in vitro models should fulfill several requisites such as recapitulate the tissue complexity - including the presence of multiple cell types/tissues, compartmentalization, existence of a circulatory system - be reproducible, easy to assemble and allow the generation of sufficient quantitative data. The use of conventional static $2 \mathrm{D}$ monolayer and $3 \mathrm{D}$ cell culture systems, to which tissue engineering significantly contributed, to assess cell-nanoparticle interaction fail to simulate in vivo conditions mainly due to the lack of dynamic flow. Besides the creation of gradients of (bio)molecules that do not reflect the in vivo conditions, static models introduce artefacts that may influence the outcomes. In the absence of flow nanoparticles can sediment, which directly impacts nanoparticle uptake by cells and thus influences the studies of nanoparticle dosage/toxicity. Recent advances in the development of dynamic bioreactors at the microscale level have positively contributed to this scenario. Commonly referred as microfluidic systems, such bioreactors allow cell, organ and even small organism cultures within controlled physiological flow and dynamic spatiotemporal controlled molecular gradients. Using such systems one can control cell-cell as well as cell-matrix interactions in addition to tissue-to-fluid 
ratio. Microfluidic devices are fabricated in a very controlled way, taking advantage of soft lithography techniques and can be easily assembled with different geometries and features to fulfill particular needs of the tissue that is being modeled. Furthermore, these systems are normally built on optically transparent materials, such as poly(dimethylsiloxane) and poly(styrene), allowing their integration with the wide range of microscopic technologies previously discussed. Although still in its infancy, tissue-like microfluidic-based platforms already proved the ability to emulate different in vivo conditions. Examples include their application to mimic vascular, cardiac, renal, pulmonary and hepatic interfaces ([22] for a review). Some of these tissue-like microfluidic platforms were already used to study nanoparticle-cell interaction $[23,24]$, as well as nucleic acid delivery [25] with predictive outcomes for the in vivo application. But their full applicability to explore and optimize drug and nucleic acid delivery systems is yet to be reached.

\section{"Visualizing' the future}

The dissection of nanoparticle-cell interaction mechanisms and the assessment of their distribution and fate at the subcellular level are of great importance since these events can give us cues about their effect/action on cells and aid in the definition of key requirements for nanoparticle design and preparation. Due to its unique features, microfluidics are more and more prominent in the nanomedicine field and in the assessment of biofunctionality of nanoparticle-based vectors. The combination of microfluidic platforms together with the application of new (quantitative) bioimaging tools will accelerate the progress of preclinical nanoparticle studies contributing to a more educated and focused advance of the field and bringing to the light of day of the long-awaited benefits.

\section{Financial \& competing interests disclosure}

The work was financed by Portuguese funds through FCT - Fundação para a Ciência e a Tecnologia in the framework of project UID/BIM/04293/2013. CDF Lopes acknowledges FCT for her PhD scholarship (SFRH/BD/77933/2011). The authors have no other relevant affiliations or financial involvement with any organization or entity with a financial interest in or financial conflict with the subject matter or materials discussed in the manuscript apart from those disclosed.

No writing assistance was utilized in the production of this manuscript. 


\section{REFERENCES}

1 Murugan K, Choonara YE, Kumar P, Bijukumar D, Du Toit LC, Pillay V. Parameters and characteristics governing cellular internalization and trans-barrier trafficking of nanostructures. Int. J. Nanomedicine 10, 2191-2206 (2015).

2 Pires LR, Oliveira H, Barrias CC et al. Imidazole-grafted chitosan-mediated gene delivery: in vitro study on transfection, intracellular trafficking and degradation. Nanomedicine 6(9), 1499-1512 (2011).

3 Iversen T-G, Skotland T, Sandvig K. Endocytosis and intracellular transport of nanoparticles: present knowledge and need for future studies. Na n o To d a y 6(2), 176-185 (2011).

4 Gao H, Yang Z, Zhang S et al. Ligand modified nanoparticles increases cell uptake, alters endocytosis and elevates glioma distribution and internalization. Sci. Rep. 3, 2534 (2013).

5 Shang L, Nienhaus K, Jiang X et al. Nanoparticle interactions with live cells: quantitative fluorescence microscopy of nanoparticle size effects. Beilstein J. Nanotechnol. 5, 2388-2397 (2014).

6 Oliveira H, Rangl M, Ebner A, Mayer B, Hinterdorfer P, Pego AP. Molecular recognition force spectroscopy: a new tool to tailor targeted nanoparticles. Small 7(9), $1236-1241$ ( 2011).

7 Pyrgiotakis G, Blattmann CO, Demokritou P. Real-time nanoparticle-cell interactions in physiological media by atomic force microscopy. ACS Sustain. Chem. Eng. 2(7), 1681-1690 (2014).

8 Hemmerich PH, Von Mikecz AH. Defining the subcellular interface of nanoparticles by live-cell imaging. PLoS ONE8 (4), e62018 (2013).

9 Peuschel H, Ruckelshausen T, Cavelius C, Kraegeloh A. Quantification of internalized silica nanoparticles via sted microscopy. BioMed Res. Int. d oi :10 .1155/ 2015 / 961208 (2015) (Epub ahead of print).

10 Graf BW, Boppart SA. Imaging and analysis of three-dimensional cell culture models. Methods Mol. Biol. 591, 211-227 (2010).

11 Böse K, Koch M, Cavelius C, Kiemer AK, Kraegeloh A. A correlative analysis of gold nanoparticles internalized by a549 cells. Part. Part. Syst. Char. 31(4), 439-448 (2014).

12 Vranic S, Boggetto N, Contremoulins V et al. Deciphering the mechanisms of cellular uptake of engineered nanoparticles by accurate evaluation of internalization using imaging flow cytometry. Part. Fibre Toxicol. 10, 2 (2013).13 Summers HD, Brown MR, Holton MD et al. Quantification of nanoparticle dose and vesicular inheritance in proliferating cells. ACS Nano 7(7), 6129-6137 (2013).

14 Garin J, Diez R, Kieffer S et al. The phagosome proteome: insight into phagosome functions. J. Cell Biol. $152(1), 165-180$ (2001).

15 Ivanov Al. Pharmacological inhibitors of exocytosis and endocytosis: novel bullets for old targets. Methods Mol. Biol. 1174, 3-18 (2014).

16 Sahay G, Kim JO, Kabanov AV, Bronich TK. The exploitation of differential endocytic pathways in normal and tumor cells in the selective targeting of nanoparticulate chemotherapeutic agents. Biomaterials 31(5), 923-933 (2010).

17 Leal C, Bouxsein NF, Ewert KK, Safinya CR. Highly efficient gene silencing activity of sirna embedded in a nanostructured gyroid cubic lipid matrix. J. Am. Chem. Soc. 132 (47), 16841-16847 (2010).

18 Chen NT, Cheng SH, Liu CP et al. Recent advances in nanoparticle-based forster resonance energy transfer for biosensing, molecular imaging and drug release profiling. Int. J. Mol. Sci. 13 (12), 16598-16623 (2012).

19 Alabi CA, Sahay G, Langer R, Anderson DG. Development of sirna-probes for studying intracellular trafficking of sirna nanoparticles. Integr. Biol. 5(1), 224-230 (2013) 
20 Shen Y, Rosendale M, Campbell RE, Perrais D. Phuji, a pH-sensitive red fluorescent protein for imaging of exoand endocytosis. J. Cell Biol. 207(3), 419-432 (2014).

21 Zhu X, Zhang L, Kao YT, Xu F, Min W. A tunable fluorescent timer method for imaging spatial-temporal protein dynamics using light-driven photoconvertible protein. J. Biophotonics 8(3), 226-232 (2015).

22 Bhise NS, Ribas J, Manoharan V et al. Organ-on-a-chip platforms for studying drug delivery systems. J. Control. Release 190, 82-93 (2014).

23 Huh D, Matthews BD, Mammoto A, Montoya-Zavala M, Hsin HY, Ingber DE. Reconstituting organ-level lung functions on a chip. Science 328 (5986), $1662-1668$ (2010).

24 Kim Y, Lobatto ME, Kawahara T et al. Probing nanoparticle translocation across the permeable endothelium in experimental atherosclerosis. Proc. Natl Acad. Sci. USA 111(3), 1078-1083 (2014).

25 Whitehead K A, Matthews J, Chang PH et al.In vitro-in vivotranslation of lipid nanoparticles for hepatocellular sirna delivery. ACS Nano 6(8), 6922-6929 (2012). 